\title{
Jugend, romantische Liebe und das gute Leben
}

\author{
Gottfried Schweiger
}

Angenommen: 31. März 2021 / Online publiziert: 26. April 2021

(C) Der/die Autor(en) 2021

Zusammenfassung In diesem Text werde ich zwei Thesen entfalten: Erstens, dass romantische Liebe Teil einer guten Jugend ist. Liebe als Teil einer guten Jugend hat mehrere Bestandteile: Jugendliche sollten romantische Liebe erleben können, wenn sie es wollen. Sie sollten das Wissen und die Fähigkeiten erlangen, romantische Liebe zu verstehen und adäquat mit den positiven Potentialen und auch den Risiken umgehen zu können. Jugendliche sollten lernen, die möglichen Enttäuschungen, die mit romantischer Liebe verbunden sein können, adäquat zu verarbeiten und darin gegebenenfalls Unterstützung erfahren. Jugendliche sollten darin befähigt werden, die sozialen und kulturellen Normen und Praktiken, die die Vorstellungen romantischer Liebe prägen, zu reflektieren und zu hinterfragen. Zweitens ist zu klären, welche moralischen Rechte und Pflichten die Eltern und staatliche Institutionen haben. Ich plädiere dafür, diese so zu verstehen, dass sie Jugendliche in der Erlangung und adäquaten Auslebung romantischer Liebe unterstützen und ihnen bei der Entwicklung dieser Fähigkeiten die notwendigen Ressourcen zur Verfügung stellen sollten.

Schlüsselwörter romantische Liebe $\cdot$ Jugend · das gute Leben

\section{Einleitung}

Romantische Liebe ist in vielfältiger Art und Weise durch kulturelle und soziale Normen und Praktiken geprägt. Es wäre also falsch anzunehmen, Menschen, insbesondere Kinder und Jugendliche, wüssten einfach, was romantische Liebe ist und wie man sich verhält, wenn man jemanden liebt. Romantische Liebe ist gleichzeitig

\footnotetext{
G. Schweiger $(\bowtie)$

Zentrum für Ethik und Armutsforschung, Universität Salzburg, Mönchsberg 2a, 5020 Salzburg, Österreich

E-Mail: gottfried.schweiger@sbg.ac.at
} 
ein hohes Gut und ein Wert, der geschätzt wird - nicht nur im subjektiven Empfinden und privaten Leben, sondern auch in ihrer öffentlichen Funktion.

Die philosophische Forschung hat sich bislang vor allem damit auseinandergesetzt, was Liebe ist, welche unterschiedlichen Arten von Liebe es gibt und welchen Wert diese haben (Grau und Smuts 2017; Maurer, Milligan, und Pacovská 2014). Wenig beachtet wurde in der Philosophie, anders als in der Psychologie - etwa prominent bei Erich Fromm (Fromm 2012) - und der angrenzenden Ratgeberliteratur, allerdings bisher, wie die Fähigkeit, zu lieben, ausgebildet werden kann und welchen Wert romantische Liebe für eine gute Jugend hat. Jugend als distinkte Lebensphase zwischen Kindheit und Erwachsenenalter ist die Phase, in der erste Versuche in romantischer Liebe gemacht, Wissen um romantische Liebe, die sie prägenden Normen und Praktiken erworben und wohl die Grundlagen für spätere, langfristige und gelungene Liebesbeziehungen gelegt werden.

Jugendliche romantische Liebe wird jedoch gesellschaftlich oft nicht primär als Chance und Entwicklungsstufe gesehen, sondern einerseits defizitär wahrgenommen („das ist noch keine echte Liebe“), andererseits gibt es einen Gefahren-Diskurs, der insbesondere die mit romantischer Liebe verbundene Sexualität als potentiell gefährlich, schuldig und unmoralisch versteht (insbesondere sexuelles Begehren von Mädchen wird negativ beurteilt) (Best und Bogle 2014). Ziel dieses Beitrags ist es, auszuloten, welche Rolle romantische Liebe als Teil eines guten Lebens von Jugendlichen spielt, welchen Wert sie hat, inwieweit Jugendliche auf romantische Liebe einen moralischen Anspruch haben können und welche Rolle die Eltern und der Staat und seine Institutionen dabei einnehmen sollten.

\section{Eine gute Jugend}

Ein Konzept einer guten Jugend ist normativ und nicht sozialtheoretisch; es beschreibt nicht Formen der Jugend in dieser oder jener Gesellschaft und auch nicht, was Jugendliche selbst unter einer guten Jugend verstehen. Vielmehr soll das Konzept einer guten Jugend skizzieren, wie Jugend durch die sie bestimmenden Institutionen gestaltet werden sollte. Jugendliche haben ein Recht auf eine gute Jugend in dem Sinne, dass die sozialen Bedingungen so eingerichtet werden sollten, dass sie Möglichkeiten vorfinden, eine gute Jugend verwirklichen zu können. Ein Konzept einer guten Jugend ist somit ein offenes Ideal und ermöglicht es, spezifische Fragen von Rechten und Pflichten einzelner Akteure und der Jugendlichen zu stellen. Was ist einer guten Jugend abträglich, was schadet ihr, welche Unterstützung benötigen Jugendliche, um eine gute Jugend als Möglichkeit vorzufinden?

Jugend als distinkte Lebensphase ist an den Rändern unscharf und die Übergänge fließend. Sie ist eine biologische Phase, die mit körperlichen und psychischen Veränderungen einhergeht, aber auch eine soziale Phase, also eine Phase, in der sich die Lebenswelt dieser jungen Menschen verändert, neue soziale Normen und Praktiken an sie herangetragen werden und sie eine ganze Reihe an neuen Erfahrungen mit sich und anderen machen.

Eine mögliche Abgrenzung gegenüber der Kindheit, die sowohl in der Biologie und Medizin als auch in den Sozialwissenschaften relevant ist, kann im Erreichen

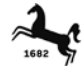


der Pubertät gesehen werden. Die Pubertät ist ein hochkomplexer körperlicher und psychischer Vorgang, der verschiedentlich sozial eingerahmt wird (Stronski 2018). Die Pubertät setzt nicht bei allen im gleichen Alter an und sie verläuft auch nicht für alle Jugendliche gleich; es gibt zum Beispiel wesentliche Unterschiede zwischen Mädchen, die tendenziell früher pubertieren, und Jungen. Dass Pubertät nicht einfach ein von sozialen Faktoren unabhängiges körperliches Geschehen ist, zeigt sich schon daran, dass in modernen Ländern aus noch nicht ganz geklärten Gründen (einen Einfluss hat wohl die Nahrungssicherheit) die Pubertät immer früher einsetzt (Drury und Bukowski 2013), und wie mit der Pubertät umgegangen wird, ist von Kultur zu Kultur unterschiedlich. Die Pubertät ist aber nur eine Möglichkeit den Anfang der Jugend zu setzen, ebenso möglich ist es, die Jugend durch Altersgrenzen festzulegen, wie das im Recht geschieht (im Jugendrecht ist man ab dem 14. Lebensjahr ein Jugendlicher) oder im Rahmen sozialwissenschaftlicher Studien, wo oft alle Teenager - als Menschen ab dem 13. Lebensjahr - als Jugendliche gelten. Diese Altersgrenzen korrelieren mehr oder weniger gut mit den körperlichen und psychischen Veränderungen und der Veränderungen der jugendlichen Lebenswelt.

Während am Anfang der Jugend, insofern die Pubertät als Anfang gesetzt wird, körperliche und psychische Veränderungen stehen, die sozial eingerahmt werden, steht an ihrem Ende vor allem die soziale Konvention einer Altersgrenze. Die Pubertät führt zur deutlich sicht- und spürbaren Ausbildung körperlicher geschlechtsspezifischer Merkmale, während der Übergang ins Erwachsenenalter sehr viel unauffälliger abläuft. Ob die Jugend daher schon mit achtzehn Jahren endet, wie dies vielfach angenommen wird und auch im Recht seinen Niederschlag findet, kann auf Basis medizinischer Erkenntnisse jedenfalls bezweifelt werden, wenn auch die körperlichen und psychischen Veränderungen weniger drastisch und auffällig stattfinden (Sawyer u. a. 2018). Gemäß den sozialen Normen und Praktiken, die die Vorstellung von Kindheit, Jugend und Erwachsenenalter prägen, ist das Ende der Jugend vor allem dadurch erreicht, dass diese Menschen selbstständig werden, für sich selbst sorgen können, alle Rechte und Pflichten von Erwachsenen genießen, aber eben auch die Verantwortung für ihr Handeln übernehmen müssen.

Eine gute Jugend wird also dahingehend zu fassen sein, dass sie diesen Veränderungen, die ihren Anfang markieren und während ihr ablaufen, berücksichtigt und auch den Blick darauf richtet, was nach ihrem Ende kommt, wenn der Jugendliche zu einem Erwachsenen geworden ist (oder zu einem solchen deklariert wurde). Der Schritt ins Erwachsenenleben ist trotz teils fixierter rechtlicher Grenzen - etwa im Wahlrecht, ausdifferenzierter dagegen im Strafrecht, welches versucht, auf die Entwicklungsstufe Rücksicht zu nehmen - als Übergang zu verstehen. Viele Dinge, die typisch sind fürs Erwachsenenleben und dort auch als normativ wertvoll verstanden werden, wie Arbeit, Familiengründung, Selbstständigkeit von den Eltern etc., treten nicht plötzlich ein, sondern werden im Laufe des Erwachsenenlebens schrittweise realisiert. Die Jugend legt dafür vielfach die Basis.

Diese starke Entwicklungskomponente gepaart mit einem Ausblick auf das Erwachsenenleben teilt die Jugend mit der Phase der Kindheit. Viel stärker als während der Kindheit - hier verstanden als Phase vor der Jugend - ist für die Jugend aber auch die Autonomie der Jugendlichen in den Blick zu nehmen und die expansive Ausweitung ihrer Lebenswelt durch die Schaffung eigener sozialer Räume und Be- 
ziehungen. Während der Jugend sind diese schon ähnlich zu den sozialen Räumen und Beziehungen von Erwachsenen - sie sind stärker geprägt durch selbstbestimmte Handlungen und reflektierte Präferenzen. Daher können Jugendliche auch schon besser und kompetenter als Kinder - instrumentelle Beziehungen eingehen und soziale Rollen annehmen und zwischen ihnen unterscheiden (wie verhalte ich mich als Schülerin, als Freundin, als Kind, als Liebhaberin). Alle diese Dinge fallen natürlich nicht einfach vom Himmel, um es kolloquial auszudrücken, sondern sind das Resultat körperlicher und damit auch psychischer Entwicklungsprozesse und sozialer Lernprozesse, die auch nicht alleine, sondern in bereits bestehenden sozialen Beziehungen geschehen (van de Bongardt u. a. 2015). Eine gute Jugend wird jedenfalls viel stärker als eine gute Kindheit von der Spannung zwischen Schutz und Autonomie geprägt, von der Eigenwilligkeit des Jugendlichen und der Begrenzung seiner Freiheit, um sein aktuelles Wohlergehen und sein Wohlentwickeln zu garantieren. Wenn auch schon kleine Kinder Anzeichen von Autonomie zeigen, so ist ihre Autonomie typischerweise weniger ausgeprägt als die von Jugendlichen. In manchen Bereichen wird man wohl davon ausgehen können, dass Jugendliche dieselbe Stufe der Autonomie erreichen wie Erwachsene, sie aber dennoch in ihren Handlungen begrenzt werden (aus paternalistischen oder anderen Gründen) (Franklin-Hall 2013; J. Anderson und Claassen 2012). Jedenfalls sind auch die Konflikte in der Jugend von anderer Qualität als in der Kindheit, da die jugendliche Widerständigkeit auf wesentlich mehr Handlungsmacht zurückgreifen kann. Es wäre ein lohnendes philosophisches Projekt eine (normative) Theorie einer guten Jugend ausgehend von psychologischen und sozialwissenschaftlichen Erkenntnissen zu entwerfen, was ich hier leider nicht leisten kann. Ich möchte aber einige Eckpunkte umreißen.

Fast alle normativ relevanten Eigenschaften, die Kinder und Erwachsene aufweisen, sind während der Jugend vorhanden, aber in veränderter Form und in Veränderung begriffen. Ich will hier nur zwei nennen: Verletzlichkeit und Autonomie. Jugendliche sind, typischerweise, noch verletzlicher als Erwachsene aber weniger verletzlich als Kinder. Natürlich gibt es innerhalb der Gruppen der Kinder, Jugendlichen und Erwachsenen jeweils Subgruppen von Menschen die verletzlicher sind, weil sie etwa chronisch krank, dement oder behindert sind. Es gibt sehr starke Kinder und sehr schwache Erwachsene, dennoch gibt es typische körperliche, mentale und auch soziale Unterschiede, die jeweils spezifische Verletzbarkeiten bedingen. Jugendliche haben stärkere Körper als Kinder und sind weniger auf Fürsorge angewiesen, sind aber gerade durch die Veränderungen der Pubertät in einer emotional und körperlich besonders vulnerablen Situation. Sie müssen mit gravierenden Veränderungen ihrer selbst zurechtkommen und sich als Individuen verstehen und positionieren lernen. Als Jugendliche werden sie (noch stärker) den sozialen Normen ausgesetzt, die an ihre Körper und Geschlechterrollen angelegt werden (Jewell und Brown 2013). Das birgt neue Verletzlichkeiten in sich, und zwar sowohl inhärente als auch situationale und gegebenenfalls pathogene (Mackenzie, Rogers, und Dodds 2014), die durch die soziale Strukturierung der jugendlichen Lebenswelt erzeugt werden. Die Ausweitung der Handlungsspielräume birgt Chancen und Gefahren gleichermaßen - Jugendliche können sich zwar sicherer im Straßenverkehr bewegen als Kinder, haben aber auch zahlreich die Möglichkeit, sich selbst und andere gerade hier zu gefährden (angetrunkenes Radfahren, Mutproben, die lega- 
le Möglichkeit, ein Moped oder gar ein Auto zu fahren etc.). Jugendliche haben qualitativ und quantitativ mehr Möglichkeiten als Kinder, sich bewusst selbst, wie auch andere zu verletzen. Verletzlichkeit und Autonomie sind somit eng verzahnt (Schweiger und Graf 2017). Während bei Kindern oft die fehlende Autonomie als Quelle für Verletzlichkeit ausgewiesen wird, zum Beispiel auch die Verletzlichkeit gegenüber der Macht ihrer Eltern (Macleod 2017), ist bei Jugendlichen, die sich immer weiter ausdehnende Autonomie gleichermaßen Schutz vor bestimmten Verletzlichkeiten als auch deren Quelle. Das ist normativ deshalb interessant, weil es zu Abwägungsfragen führt, etwa inwieweit jugendliche Autonomie autoritativ ist, wenn es um Risiken und Gefahren geht, die der Jugendliche eingehen will oder vielleicht auch sollte, um andere Güter zu erreichen. Etwa das Risiko einer Operation, um gesund zu werden. Oder das Risiko, die Schule frühzeitig zu verlassen und stattdessen zu arbeiten. Es bedarf hier der Entwicklung von Kriterien, wann jugendliche Autonomie als soweit ausgereift gelten kann, dass sie jener von Erwachsenen entspricht und dann aber auch der Entwicklung von Kriterien, wann jugendliche Autonomie, sowohl solche, die Erwachsenen gleichwertig ist, als auch solche, die noch unreife Autonomie ist, bei der Entscheidung über die Zulässigkeit einer Handlung Autorität beanspruchen kann. Mit unreifer Autonomie ist hier gemeint, dass Kinder und Jugendliche über manche Eigenschaften verfügen, die Autonomie auszeichnen, aber eben noch nicht über alle. Sie können zum Beispiel zwar Wünsche formulieren, aber die Konsequenzen noch nicht ausreichend bedenken. In einigen Fällen ist diese unreife Autonomie ausreichend, da die Entscheidungen, um die es geht, nicht komplex oder folgenschwer sind. Eine Jugendliche kann gut darüber entscheiden, welchen Kinofilm sie sich ansehen möchte, aber sie ist noch nicht in der Lage, wohlüberlegte Entscheidungen als Bundeskanzlerin zu treffen.

Es sind nämlich nicht nur Fälle denkbar, in denen paternalistisch gegen die vorhandene und Erwachsenen gleichwertige jugendliche Autonomie zu entscheiden ist, sondern auch solche, in denen trotz nicht oder nur unreif vorhandener jugendlicher Autonomie ihnen die Entscheidung überlassen werden sollte. Der letzte Fall ist auch für Kinder interessant, da er darauf abzielt, dass in manchen Fällen dem Willen Autorität zukommen sollte, auch wenn keine oder nur unreife Autonomie vorhanden ist. Monika Betzler hat etwa dafür plädiert, dass die Erziehung zur Autonomie verlangt, dass Kinder Autonomie einüben können, was bedeutet, ihnen immer wieder (ungefährliche) Entscheidungen zu überlassen, auch wenn sie dafür noch nicht vollständig kompetent sind (Betzler 2011). Man könnte hier aber auch an den Fall eines Wahlrechts für sehr junge Kinder denken, da für dessen Ausübung aus demokratiepolitischen Gründen gar keine bestimmten Fähigkeiten verlangt werden. Die arbiträre Altersgrenze des Wahlalters scheint aus diesen Gründen unfair, da sie nicht sachlich - mit Blick auf Autonomiekompetenzen - begründet wird (Merk 2009; kritisch dazu: Giesinger 2017).

Eine gute Jugend wird sich also zwischen diesen Polen des Schutzes und der Ermöglichung von Autonomie bewegen und Wert auf die Entfaltungsmöglichkeiten von Jugendlichen legen, die es ihnen erlauben, ihre eigene Identität auszubilden und auch in vielen Lebensbereichen, sich selbst zu verwirklichen. Auch eine gute Kindheit ist zwischen diesen Polen aufgespannt (Betzler 2011), doch wird in der Jugend immer stärker die Autonomie, der Selbstverwirklichung und die Frage der 
Identität in den Vordergrund kommen. Dieser Selbstverwirklichung sind Grenzen gesetzt, die sich aus unterschiedlichen Quellen ergeben. Eine Grenze wird durch die Selbst- und Fremdgefährdung markiert. Die Selbstgefährdung ist aber enger zu fassen als bei Erwachsenen und zwar nicht nur aus Gründen eingeschränkter Autonomie, sondern auch aus Gründen der Etablierung eines Schutzraums der Jugend, wie ihn Joel Anderson und Rutger Claassen beschreiben (J. Anderson und Claassen 2012). Ein solcher Schutzraum ist eben auch aus sozialen Gründen konstituiert, er soll Jugendlichen die Möglichkeit geben, sich selbst zu finden und Dinge auszuprobieren, ohne dafür die volle Last der Konsequenzen tragen zu müssen. Dahingehend dient diese Grenze der jugendlichen Autonomie dann nicht nur dem Schutz vor Gefahren, sondern auch um Erlebnis- und Lernräume zu schaffen. Von einem Schutzraum zu sprechen ist hier aus zwei Gründen sinnvoll. Jugendliche werden vor Gefahren von außen geschützt, in dem sie bestimmte Dinge nicht tun dürfen, zum Beispiel solche Medien zu konsumieren, die ihre Entwicklung schädigen oder langfristige Entscheidungen zu treffen, die sie noch nicht abschätzen können, wie eine Heirat oder einen hohen Kredit aufzunehmen. Sie sollen auch vor Erwachsenen geschützt werden, die ihre jugendliche Verletzlichkeit ausnützen. Der Schutzraum ist aber auch darauf ausgelegt, dass Jugendliche noch nicht für sich selbst sorgen müssen und in ihrer Entwicklung von anderen unterstützt werden. Der Schutzraum ist natürlich idealisiert und in vielen Ländern, und in Ausnahmesituationen auch in Deutschland und Österreich, sind Jugendliche alleine auf der Straße und leben ein Leben wie Erwachsene. Dieser Schutzraum dient schließlich auch dazu, allen Jugendlichen, die ja trotz gleichen Alters sehr unterschiedlich in ihren Fähigkeiten sein können, dieselben Rechte und Pflichten zuzugestehen, ohne auf Einzelfallprüfungen der Autonomiefähigkeiten zurückgreifen zu müssen (Altersgrenzen sind oft danach strukturiert, zum Beispiel das Verbot alkoholische Getränke zu konsumieren).

Für eine gute Jugend spielen nun eine ganze Reihe an Dingen eine Rolle. Es geht um die Entwicklung von Fähigkeiten, um als Jugendliche und im späteren Leben als Erwachsener handeln zu können, den Erwerb von Wissen und wie man mit ihm produktiv umgehen kann. Es geht darum, Erfahrungen zu machen und sich ausprobieren zu können, sich mit sich selbst auseinanderzusetzen und zu beginnen, die eigene Biographie zu schreiben und das Leben langsam in die eigene Hand zu nehmen, Beziehungen aufzubauen und Entscheidungen mit größerer Tragweite zu treffen. Die psychologische und sozialwissenschaftliche Jugendforschung hat es zur Aufgabe, die Lebenswelten und die dahinter liegenden körperlichen, psychischen und sozialen Prozesse aufzuklären, während es normativ darum geht, herauszufiltern, was davon einen solchen moralischen Wert hat, dass es auf der einen Seite bei Eltern, Staat und anderen Akteuren Verpflichtungen und Verantwortung erzeugt und auf der anderen Seite spezifische Rechte von Jugendlichen begründen kann. Man könnte hier versuchen, eine Liste an Gütern oder Fähigkeiten zu entwickeln, die eine gute Jugend ausmachen und auf die Jugendliche moralischen Rechte haben. Das will ich hier aber nicht versuchen, sondern mich auf romantische Liebe als ein Gut einer guten Jugend zu konzentrieren. Aber warum sollte romantische Liebe als ein normativ wichtiger Bestandteil einer guten Jugend verstanden werden? 


\section{Eine gute Jugend und die romantischen Liebe}

Romantische Liebe ist für ein solches, hier nur sehr lose skizziertes Konzept einer guten Jugend von dreifacher Bedeutung: Als erlebte romantische Liebe, als zu lernende romantische Liebe und als Basis für die künftige romantische Liebe im Erwachsenenleben. Es geht also darum, ob und wie romantische Liebe in einer guten Jugend präsent ist, welche Lernprozesse dafür nötig sind, damit sie gelingen kann und welche Rolle die romantische Liebe während der Jugend für künftige romantische Liebe im Erwachsenenalter spielt. Um diese drei Aspekte weiter unten zu klären, möchte ich vier Vorbemerkungen machen.

Erstens stellt sich die Frage, was romantische Liebe überhaupt ist. In der philosophischen Literatur herrscht darüber keine Einigkeit (Kühler 2019), vielmehr denke ich, dass romantische Liebe ein ,essentially contested concept“ (Gallie 1956) ist und bleiben wird, also ein Konsens darüber nicht erzielt werden kann, und die Klärung dieser Frage ist auch nicht mein Interesse in diesem Aufsatz. In einem möglichst weiten Verständnis, welches für meine Zwecke ausreichend ist, ist romantische Liebe zunächst eine Emotion, die sich in starken Gefühlen für einen oder mehrere andere Menschen ausdrückt und Einstellungen der Wertschätzung, der Sorge und des sexuellen Begehrens beinhaltet. Wer jemanden romantisch liebt, will in der Regel mit dieser Person eine Beziehung eingehen und von dieser Person ebenfalls geliebt werden. Eine exakte Abgrenzung zwischen romantischer Liebe und anderen Emotionen und Nahebeziehungen wie der Freundschaft ist begrifflich und empirisch schwierig. Der Freundschaft scheint es an Exklusivität, Intensität und dem sexuellen Begehren zu mangeln. Nochmals davon zu unterscheiden ist die elterliche oder geschwisterliche Liebe. Der Liebesbegriff ist auch deshalb umstritten, weil er stark sozio-kulturell geprägt ist (Lindholm 2006), aber selbst wenn romantische Liebe, wie sie in der westlichen Welt verstanden wird, ein kontingentes sozio-kulturelles Produkt wäre, bedeutet das nicht, dass sie für die Menschen, die dieses Ideal oder diese Vorstellung von romantischer Liebe verfolgen und bejahen, nicht für ein gutes Leben relevant ist.

Zweitens scheint es mir, da auf der Hand liegend, nicht nötig, entsprechende Jugendstudien ausführlich zu zitieren, um zu zeigen, dass Jugendliche romantische Liebe wertschätzen und viele von ihnen diese gerne erleben (möchten) (Calmbach u. a. 2016). Das ist trivial anzunehmen in einer Welt, in der an allen Ecken mediale Liebesdarstellungen von Jugendlichen vorhanden sind und Jugendliche sehr oft Liebesbeziehungen eingehen. Dass jugendliche romantische Liebe schätzen und gerne erleben (wollen) alleine ist ein wichtiger Grund, romantische Liebe als Teil einer guten Jugend anzusehen. Ich möchte dafür auf eine Argumentation von Angelika Krebs zurückgreifen (Krebs 2001). Sie stellt sich die Frage, warum Erwerbsarbeit nicht substituierbar durch ein Grundeinkommen ist und es deshalb ein Recht auf Arbeit geben sollte. Ihr Kernargument lautet, dass das Recht auf Arbeit darauf beruht, dass wir in einer Arbeitsgesellschaft leben, also Arbeit und Anerkennung eng verknüpft sind und so lange dies der Fall ist, haben Menschen ein Recht darauf, an der Erwerbsarbeit teilhaben zu können, um eben jene Güter wie Anerkennung erfahren zu können. Außerhalb einer Arbeitsgesellschaft wäre ein Grundeinkommen eine hinreichende Alternative. In diesem Sinne gehe ich davon aus, dass romanti- 
sche Liebe ein wichtiger Bestandteil einer guten Jugend, ja eines guten Lebens ist, so lange wir in einer Gesellschaft leben, in der romantische Liebe ein so großer Stellenwert zukommt wie in der unsrigen. Es mag also vorstellbar sein, dass Gesellschaften existieren, in denen romantische Liebe kein Wert zugesprochen wird, ja in der das Konzept mehr oder weniger unbekannt ist und dann wird sie vielleicht auch nicht Teil einer guten Jugend oder eines guten Lebens als Erwachsener in eben dieser Gesellschaft sein.

Neben diesem Argument, welche romantische Liebe als wertvoll auf Basis geteilter sozialer Werte und Normen versteht, ließen sich auch Argumente vorbringen, die romantischer Liebe einen universalen normativen Wert zuschreiben. Sie hier im Detail zu explizieren, würde den Rahmen allerdings sprengen. Es ist plausibel, einen überlappenden Konsens anzunehmen, dass romantische Liebe Teil einer guten Jugend ist, der aus unterschiedlichen ethischen, religiösen oder kulturellen Gründen geteilt wird. Es lassen sich nämlich gute Gründe dafür vorbringen. Nicht nur wird Liebe in vielen Kulturen geschätzt, sie hat viele positive Eigenschaften - und birgt einige Gefahren für das physische und psychische Wohlergehen -, die in der Psychologie der Liebe gut erforscht sind und die auch für Jugendliche und ihre Entwicklung gelten (Collins 2003; Collins u. a. 2009): romantische Liebe kann Glücksgefühle auslösen, Zufriedenheit, Geborgenheit und Vertrauen. Durch romantische Liebe werden Erfahrungen mit sich selbst und anderen möglich, nämlich tiefe und enge Beziehungen, die ohne sie nicht existieren würden. Darin unterscheidet sich romantische Liebe auch von anderen Emotionen, Wünschen und Handlungen. Nicht alles, was Jugendliche wollen, ist ihnen zu erlauben oder gar zu ermöglichen, aber die romantische Liebe scheint mir dafür qualifiziert zu sein, ein solches Gut zu sein, dass sie nicht ohne gute Gründe beschränkt werden darf und dass ihr Erlebnis so wichtig und positiv ist, dass Jugendliche in Bezug auf sie bestimmte moralische Rechte haben - wobei ich unten noch erläutern werde, worauf Jugendliche hier genau Rechte haben, wofür es wichtig ist, unterschiedliche Aspekte der romantischen Liebe zu differenzieren. Diese und andere positive Eigenschaften von romantischer Liebe wurden in unterschiedlichen philosophischen Theorien akzentuiert, ihr Kern bleibt aber darauf verwiesen, dass Menschen eben diese Erfahrungen machen und diese Gefühle haben und als wertvoll empfinden.

Drittens ist das Faktum, dass Jugendliche zur romantischen Liebe fähig sind, wichtig. Könnten nur Erwachsene romantische Liebe empfinden, dann wäre diese nicht Teil einer guten Jugend. Anzuerkennen, dass Jugendliche Liebe erfahren und ausleben können, hat nämlich zumindest zur Folge, dass die Eltern oder der Staat darauf reagieren müssen. Das eröffnet die Frage, wie sie darauf reagieren sollen, etwa indem sie nichts tun, die Jugendlichen unterstützen oder mit Sanktionen reagieren. Liebeshandlungen zu verbieten und Jugendliche dahingehend zu kontrollieren, bedarf zumindest der Rechtfertigung, vor allem insofern man davon ausgeht, dass romantische Liebe zwischen Erwachsenen legitim ist, was mir niemand ernsthaft $\mathrm{zu}$ bestreiten scheint. Ich sehe keine überzeugenden Gründe Jugendlichen prima facie zu untersagen, sich zu verlieben, sich lieben zu lassen oder eine Liebesbeziehung einzugehen. Bestimmte Liebeshandlungen können und sollen verboten werden, wenn sie zu gravierenden Gefährdungen des Wohlergehens der Jugendlichen führen, aber romantische Liebe per se fällt hier nicht darunter. Daraus lässt sich zumindest 
folgern, dass Jugendliche ein Recht darauf haben, dass etwaige Einschränkungen ihrer romantischen Liebe ihnen gegenüber begründet werden. Selbst wenn man also, obwohl das nicht plausibel scheint, davon ausgeht, dass romantische Liebe nicht Teil einer guten Jugend ist, so wird man anerkennen müssen, dass sie Teil der gelebten Jugend ist und auch in diesem Fall sind viele der nachfolgenden Reflexionen von Bedeutung, weil Jugendliche auch lernen müssen, mit Dingen umzugehen, mit denen sie vielleicht nicht umgehen sollten, es aber, aus welchen Gründen auch immer, tun.

Viertens ist es sinnvoll romantische Liebe in die von mir genannten Dimensionen zu differenzieren, da damit unterschiedliche normative Fragen verbunden sind. Wenn von Liebe als Emotion die Rede ist, stellt sich zum Beispiel die Frage des Werts und der Regulierung anders als wenn von romantischer Liebe als einer gelebten Beziehung gesprochen wird. Romantische Liebe als Emotion lässt sich nicht verbieten, es lässt sich nur der Umgang mit ihr erlernen und sie lässt sich reflektieren. Romantische Liebe als gelebte Beziehung mit anderen Menschen wiederum ist für Eingriffe von dritter Seite, also zum Beispiel den Eltern oder dem Staat, zugänglich. Es wäre somit auch denkbar, dass es Teil einer guten Jugend ist, Liebe zwar fühlen, aber nicht ausleben zu dürfen - das scheint mir unplausibel, solange nicht Gründe dafür vorgebracht werden, warum das Ausleben romantischer Liebe das Wohlergehen der Jugendlichen oder anderer Personen oder sonstige wichtige Rechte und Pflichten verletzt. Dann sind hier natürlich auch unterschiedliche Dinge, die es zu lernen gilt: den Umgang mit eigenen Emotionen zu lernen ist wichtig, auch wie man sie ausdrückt und wie man sich zu ihnen verhält und wie man sie in Handlungen umsetzt (oder eben nicht). Das Liebeshandeln in einer Beziehung wiederum ist sehr viel komplexer als der Umgang nur mit eigenen Emotionen, was sich schon daran zeigen lässt, dass hier eine zweite Person - oder mehrere Personen - unmittelbar beteiligt sind. Es sind also diese unterschiedlichen Dimensionen der romantischen Liebe als Teil einer guten Jugend, die ich in den folgenden Abschnitten beleuchten will.

\section{Jugend und das Erlebnis der romantischen Liebe}

Mit der Erlebnisdimension der romantischen Liebe will ich sagen, dass es Teil einer guten Jugend ist, romantische Liebe erleben zu können, wenn man es will. Es mag sein, dass nicht jeder Jugendliche in seiner Jugend verliebt ist und sicherlich sind nicht alle glücklich verliebt oder genießen glückliche Beziehungen. Das ist im Fall von Erwachsenen auch nicht anders. Es mag auch Menschen geben, sowohl Jugendliche als auch Erwachsene, die dies nicht als Mangel erleben, sei es, weil sie freiwillig darauf verzichten wollen oder weil sie auf Grund vorliegender psychischer Krankheiten nicht dazu in der Lage sind, Liebe oder andere Emotionen zu empfinden. Diesen Menschen gegenüber wäre es unfair, wenn ihnen gesagt werden würde, ihr Leben wäre weniger gut, weil es frei von romantischer Liebe ist, obwohl ihnen natürlich Hilfe angeboten werden sollte, wenn sie es wünschen. Daher ist es auch so wichtig, die Erlebnisdimension als eine Option zu verstehen. Eine gute Jugend besteht also darin, die Möglichkeit zu haben, romantische Liebe zu erfah- 
ren, wenn man diese Option wünscht, und diese Jugend wird dadurch schlechter, wenn sie unfreiwillig nicht vorhanden ist. Daraus folgt noch nicht, dass Jugendliche oder Erwachsene ein Recht darauf haben, geliebt zu werden oder dass ihre Liebe erwidert wird. Die Erlebnisdimension ist mit vielfältigen Emotionen, Präferenzen und Handlungen verknüpft. Romantische Liebe kann im stillen Kämmerlein bei der Niederschrift romantischer Gedichte erlebt werden, beim Candle Light Dinner zu zweit oder auch bei der Versendung romantischer oder erotischer Textnachrichten.

Daher ist die Erlebnisdimension mindestens in zwei Komponenten aufzuspalten: auf der einen Seite die Innenseite, also das Erlebnis von romantischer Liebe als Innenerlebnis, was oft als das Erleben einer Emotion verstanden wird, auf der anderen Seite die Außenseite, also das Erlebnis von romantischer Liebe als komplexes Beziehungsgeschehen und -handeln, welches mit einer ganzen Reihe an Symbolen und materiellen Dingen verknüpft ist. Oft fallen beide zusammen, aber natürlich kann es sie auch getrennt voneinander geben. Man kann romantische Liebe fühlen, ohne Liebeshandlungen zu setzen, und man kann Liebeshandlungen setzen, ohne aber dieses Gefühl zu erleben. Beides ist vielleicht defizitär, jedoch aus anderen Gründen. Die Erlebnisdimension romantischer Liebe in der Jugend ist stark kulturell geprägt, folgt auch individuellen Präferenzen und ist auch durch die Situation der Jugend eingeschränkt. Das betrifft vor allem die Erlebnisdimension als ein Set von Handlungen.

Letzteres, also die spezifische (soziale) Situation der Jugend, scheint mir hier normativ nochmals wichtig zu betonen, weil es den entscheidenden Unterschied zur romantischen Liebe im Erwachsenenalter markiert. Jugendliche können sehr viele Dinge tun, die Erwachsene tun können, viele Dinge aber auch nicht. Für viele Erwachsene gehört es zur romantischen Liebe dazu, zu heiraten, zusammen zu wohnen oder eine Familie zu gründen. Diese Dinge sind Jugendlichen größtenteils verwehrt bzw. werden ihnen erschwert und sie werden aktiv dazu angehalten, sie nicht zu tun, auch wenn sie dies könnten. Es ist natürlich nicht so, dass die meisten Jugendliche hier gegen ihren Willen von einer Hochzeit, dem Zusammenziehen oder Kindern abgehalten werden müssen, sondern viele wollen dies gar nicht, auch wenn es wohl nur schwer feststellbar ist, inwiefern diese Präferenzen nicht selbst das Resultat sozialer Normen und Praktiken und ihrer Erlernung sind - das trifft aber bei Erwachsenen auch so zu. Jugendlichen diese Dinge, die als Liebeshandlungen verstanden werden können, nicht zu erlauben (wobei die Verbote hier unterschiedlich gehandhabt werden), fußt sowohl auf Überlegungen hinsichtlich des Schutzes ihres Wohlergehens als auch ihres Wohlentwickelns, wobei das Wohlentwickeln hier eng verknüpft ist mit der Annahme, ihnen eine möglichst offene Zukunft zu ermöglichen (Mills 2003). Eine frühe Heirat oder gar eigene Kinder können Pfadabhängigkeiten schaffen, die nur schwer korrigiert werden können. Sie legen aber sicherlich vor allem dem Leben während der Jugend Lasten auf, die Jugendliche ohne Unterstützung durch Erwachsene oder Institutionen alleine nicht einfach bewältigen können. Das hat auch mit der Einrichtung moderner Gesellschaften zu tun: weil in Wissensgesellschaften Bildungsabschlüsse für den sozioökonomische Position so wichtig sind (und diese wiederum so wichtig für das Wohlergehen in einer kapitalistisch verfassten Gesellschaft), ist die Ausbildung während der Jugend und im Anschluss darauf aufbauend wichtig. Jugendliche, die zugunsten früher Elternschaft 
auf Ausbildung verzichten, sind also für ihren gesamten Lebenslauf benachteiligt, insbesondere wenn die staatlichen Strukturen oder persönliche Netzwerke fehlen (Cook und Cameron 2015).

Die Erlebnisdimension von romantischer Liebe in der Jugend ist also anders als die von Erwachsenen. Sie ist, so könnte man vereinfacht sagen, weniger stark durch soziale Verpflichtungen geprägt, die sich etwa im Zusammenleben, der Rücksichtnahme auf wichtige Lebensentscheidungen (zum Beispiel bei der Wahl des Arbeitsund Wohnorts), der Familienplanung oder der finanziellen Verwobenheit in vielen erwachsenen romantischen Liebesbeziehungen ausdrücken. Der Staat, der bestimmte Formen der Beziehung wie die Ehe privilegiert und unterstützt, tut hier etwas dazu, solche Bindungen in romantischen Partnerschaften zu stabilisieren, es kann aber wohl auch davon ausgegangen werden, dass viele Erwachsene sich eng aneinander binden wollen. Natürlich können auch Erwachsene verliebt sein und in Beziehungen leben, wo es sehr wenige solcher Verpflichtungen gibt (getrennter Haushalt, keine Kinder, getrennte Kassen). Das ändert aber nichts daran, dass man erwarten würde, dass manche Verpflichtungen dennoch stärker vorhanden sind als während der Jugend. Im Erwachsenenalter wird es wohl zu einer Liebesbeziehung gehören, dass man den Partner im Fall einer Krankheit nicht nur emotional unterstützt, sondern auch, wenn möglich, finanzielle und pflegende Verantwortung übernimmt, wie das etwa Philipp Pettit als robuste Sorge umschreibt (Pettit 2015). In vielen Fällen schon deshalb, weil bei Erwachsenen diese Fürsorge nicht mehr durch die eigenen Eltern geleistet wird, während bei Jugendlichen man solche Verantwortungsübernahme durch den anderen jugendlichen Liebespartner nicht erwarten wird, ja, es sogar eine moralische Überforderung wäre, wenn das nötig wäre. Auch jugendliche Liebespartner sollten für einander da sein, wenn es schwere Zeiten gibt, aber diese Verpflichtungen sind stark abgeschwächt.

\section{Lernprozesse romantischer Liebe}

Romantische Liebe soll während der Jugend aber nicht nur erlebt werden, sondern sie soll auch erst einmal erlernt und eingeübt werden. Hier sind mehrere miteinander verwobene Dimensionen zu unterscheiden. Die komplexe emotionale Dimension der romantischen Liebe erfordert, dass man sich zu ihr verhalten kann. Sie werden nicht nur gefühlt, sondern auch reflektiert. Das ist jedoch nicht einfach gegeben, sondern basiert auf Lernprozessen und verlangt das Erreichen eines bestimmten geistigen Niveaus. Kinder können zwar Zuneigung spüren und auch ausdrücken, die komplexe Emotion der romantischen Liebe ist ihnen aber fremd, obwohl sie wahrscheinlich schon Vorformen davon erleben können und frühkindliche Erfahrungen einen Einfluss auf das spätere Erleben romantischer Liebe haben können - das wird sowohl aus neurobiologischer als auch bindungstheoretischer und psychoanalytischer Sicht festgehalten (Walter 2004; Neumann 2002). Prominent in die Philosophie eingebracht hat die Bedeutung frühkindlicher Liebeserfahrung für die weitere Identitätsentwicklung Axel Honneth im Rahmen seiner Theorie der Anerkennung (Honneth 1992). Auch aus dieser Perspektive der miteinander verwobenen Anerkennungserfahrungen ist klar, dass frühkindliche Erfahrungen von elterlicher Liebe einen großen Einfluss 
auf die spätere, in der Jugendzeit aufbrechende, romantische Liebe und den Umgang mit ihr haben. Es macht für mich dabei auch keinen wesentlichen Unterschied, ob romantische Liebe als Emotion oder als Syndrom verstanden wird (Pismenny und Prinz 2017). Das sind wichtige psychologische und ontologische Fragen, die an der Erlebnisdimension und der Lerndimension aber nur sehr wenig ändern. Der Umgang mit romantischer Liebe, egal ob verstanden als Emotion oder als Syndrom, ist immer für den Liebenden eine Herausforderung, mit der er umgehen lernen muss, und ihr Ausdruck in Handlungen und Symbolen ist immer sozial geprägt.

Es lassen sich für die Lerndimension der Liebe also zumindest zwei wichtige Dimensionen unterscheiden: Auf der einen Seite steht der Lernprozess, die Innenseite der romantischen Liebe, die in einem selbst verortet ist, zu verstehen und sich damit auseinanderzusetzen. Hier kann das Konzept der epistemischen Gerechtigkeit weiterhelfen, vor allem jenes der hermeneutischen Gerechtigkeit, die von Miranda Fricker so beschrieben wird, dass Menschen in die Lage versetzt werden, sich selbst und ihr Innenleben $\mathrm{zu}$ verstehen und anderen auch davon $\mathrm{zu}$ verstehen $\mathrm{zu}$ geben (Fricker 2007). Ihr Beispiel einer hermeneutischen Ungerechtigkeit ist dasjenige, eines homosexuellen Menschen, der in einer Gesellschaft lebt, die Homosexualität scharf verurteilt und als Sünde ansieht, und dadurch nicht die Mittel an die Hand bekommt, sich selbst, die eigene Gefühlswelt und die eigenen sexuellen Präferenzen adäquat zu verstehen. Auch das gehört aber zum Erlernen romantischer Liebe dazu, nämlich die sexuellen Vorlieben und Neigungen, die in der romantischen Liebe ja eine wichtige Rolle spielen, adäquat verstehen zu lernen, die kommunikativen Mittel und einen Raum vorzufinden, in dem man diese für sich selbst und für andere artikulieren kann.

Auf der anderen Seite der Lerndimension steht die Außenseite der romantischen Liebe, die sich in Handlungen, Beziehungen, Symbolen und Dingen ausdrückt. Eva Illouz hat eindrücklich gezeigt, dass Liebeshandeln im Kapitalismus nicht nur konventionell gebunden ist, sondern auch stark mit Dingen und Symbolen verknüpft ist (Illouz 2009; 2007). Beispiele dafür sind die roten Rosen, die man sich schickt, das Candle Light Dinner, zu dem man gemeinsam geht oder auch die Reizwäsche, die man für den anderen trägt. Diese Außenseite ist hoch komplex, kulturell differenziert und im historischen Wandel der Liebeshandlungen schon innerhalb weniger Generationen stark verändern kann. Dazu tragen nicht nur veränderte soziale Normen bei, sondern auch neue Techniken, man denke nur an die Möglichkeiten des Liebeshandeln über das Internet mit seinen Partnervermittlungsportalen, Messengerdiensten und sozialen Medien (Ben-Ze'ev 2004). Damit umzugehen, muss erlernt und ein Stück weit immer auch ausprobiert werden. Schließlich sollen die Handlungen ja nicht nur gemäß Konventionen gesetzt, sondern auch inkorporiert werden, wozu man ihren symbolischen Gehalt verstehen muss. Das Ziel ist ja nicht, dass sich die Partner rote Rosen schenken und dabei nichts fühlen oder es nicht verstehen, sondern, dass sie dies tun und dadurch ihrer romantischen Liebe Ausdruck verleihen und Gefühle erzeugen bzw. verstärken. Die Außenseite der romantischen Liebe ist schon deshalb von so großer Bedeutung, weil über sie die Innenseite erst $\mathrm{zu}$ einem sozialen Geschehen werden kann und von der bloßen Liebe zu einem anderen Menschen zu einer Liebesbeziehung, die meistens angestrebt wird. Es ist auch davon auszugehen, dass die Außenseite sich auf die Innenseite zurückbiegt und 
diese wiederum beeinflusst. In der Außenseite der romantischen Liebe, also ihrer Handlungsdimension, liegen auch die Risiken und Gefahren, die oft im Vordergrund elterlicher und staatlicher Aufmerksamkeit stehen.

Einerseits wird das Liebeshandeln beschränkt, wie oben schon erwähnt wurde. Jugendliche sollen (und dürfen auch aus Sicht der Eltern oder gesetzlicher Regeln) nicht alle Liebeshandlungen setzen, die Erwachsene dürfen. Oder sie dürfen manche Liebeshandlungen, wie Sex, nur mit anderen Jugendlichen, aber nicht mit Erwachsenen setzen. Das ist auch zu lernen und diese Grenzen sollten verständlich gemacht werden, warum es sie gibt und welchen Nutzen sie für den Jugendlichen haben. Es gibt auch Liebeshandlungen, die sowohl für Erwachsene als auch Jugendliche als schädlich einzustufen sind und eine Intervention durch Dritte rechtfertigen, ja sogar moralisch gebieten, wie zum Beispiel Selbstverletzungen aus Liebeskummer. Goethes Werther ist ein literarisches Beispiel für die tragischen Folgen, die Liebeskummer haben kann.

Andererseits sind auch solche Liebeshandlungen, die für Jugendliche erlaubt sind und die mitunter auch als positiv angesehen werden, mit Risiken verbunden. Im Fokus steht hier oft die mit romantischer Liebe verbundene Sexualität, die mitunter das Ausmaß einer moralischen Panik annehmen kann, wie der Diskurs um Teenagerschwangerschaften in den USA oder in England zeigt (Selman 2003). Es geht hier also etwa um das Risiko von sexueller Gewalt in romantischen Liebesbeziehungen, das Risiko sexuell übertragbarer Krankheiten, das Risiko einer (ungewollten) Schwangerschaft oder das Risiko, sexuelle Handlungen zu setzen, die später bereut werden. In Zeiten des Internets und der Smartphones sind solche Handlungen wie Sexting, also das Verschicken von erotischen Fotos und Videos, oder das (unerwünschte) Hochladen solchen Materials auf Pornowebseiten als Risiken neu hinzugekommen (Dekker und Thula Koops 2017). Risiken sind real vorhanden und romantisches Liebeshandeln ist eingelassen in Machtverhältnisse (Gregoratto 2017) und Jugendliche sollten lernen, sich damit auseinanderzusetzen. Sexualität besonders, aber sicher nicht nur sie, weist eine enge Verknüpfung mit Geschlechterrollen auf und ist stark durch soziale sowie moralische und kulturelle Normen geprägt (Gill 2012).

Bislang wurde von mir auch das Verhältnis von romantischer Liebe und Autonomie noch nicht hinsichtlich seiner Konfliktpotentiale beleuchtet. Liebe kann nämlich nicht nur Verwirklichung der Autonomie bedeuten, sondern auch eine Beschränkung (Lehrer 1997). Einerseits durch Tendenzen, den anderen besitzen und an sich binden zu wollen - das kann durch soziale und kulturelle Normen sogar in extremer Form wie der rechtlichen Verfügungsmacht von Männern über Frauen legitimiert werden. Andererseits kann Liebe aber auch zur Selbstaufgabe führen, also zur Einschränkung der eigenen Autonomie, weil man den anderen liebt. Diese Einschränkung aus Liebe kann gewollt, erfüllend und moralisch richtig sein, sie kann aber auch verzerrt, aus der Angst vor Einsamkeit, falschen Motiven oder angelernten Rollenbildern erfolgen.

Hier gibt es also eine ganze Reihe an Wissensbeständen, die Jugendliche erwerben sollten, um romantische Liebenshandlungen überhaupt setzen zu können und vor allem auch, um diese reflektieren und auf die eigene Identität, die eigenen Wünsche und Vorstellungen beziehen zu können. Denn das ist ja schließlich, was wohl 
für ein gutes Leben (in der Jugend und als Erwachsener) zählt, nämlich nicht nur über soziale Normen und Praktiken Bescheid zu wissen, sondern sich gemäß eigenen reflektierten Wünschen und Vorstellungen authentisch selbstverwirklichen zu können. Es darf bezweifelt werden, dass dies einer großen Mehrheit (der Erwachsenen und Jugendlichen gleichermaßen) auch wirklich gelingt und es ist schwierig, hier sinnvolle normative Maßstäbe zu entwickeln, wie viel Selbstverwirklichung nötig ist für ein gutes Leben und wann diese auch erreicht wird (Fenner 2015; Abad 2014). Die kritischen Instrumente, um echte von unechter Selbstverwirklichung zu unterscheiden, etwa das Konzept der Entfremdung (Jaeggi 2005) oder adaptiver Präferenzen (Khader 2011), sind in ihrer analytischen Anwendung mit Schwierigkeiten behaftet; in ihrer empirischen Anwendung auf solche Phänomene wie romantische Liebe sowieso. Es wird dennoch eine Aufgabe bleiben, zumindest zu versuchen, Jugendlichen und auch Erwachsenen die Mittel zur Verfügung zu stellen und sie dabei zu unterstützen, für sich selbst herauszufinden, was sie wirklich wollen, warum sie dies wollen und auch, wie sie dies umsetzen wollen. Und zwar inmitten der Paradoxien moderner Gesellschaften, in denen alle Emotionen, Gefühle und Werte auch in Gefahr stehen kommerzialisiert und entfremdet zu werden (Honneth 2002). Für solche kritischen Lernprozesse gibt es auch eine Reihe an sinnvollen pädagogischen Konzepten der Wirksamkeit, die hier aber nicht diskutiert werden können (Helmer u. a. 2015).

\section{Liebe während der Jugend und im Erwachsenenleben}

Sowohl die Innenseite als auch die Außenseite der romantischen Liebe, die ich oben schon in den beiden Erlebnisdimensionen angesprochen habe, haben wiederum einen Aspekt des Jetzt und einen der Zukunft. Das bringt mich zur dritten Dimension romantischer Liebe in der Jugend, nämlich den Ausblick auf die künftige Liebe im Erwachsenenalter. Mit diesem Ausblick auf die Liebe im Erwachsenenleben soll die romantische Liebe während der Jugend nicht abgewertet werden, sondern vor allem auch der Hinweis darauf erfolgen, dass sich Liebe verändert. Es verändert sich sowohl ihre Innen- als auch ihre Außenseite. Die Innenseite verändert sich im Rahmen längerer Liebesbeziehungen, durch neue Erfahrungen und Lebensumstände und in Liebesbeziehungen, die zu Familien mit Kindern werden, kommen ebenso ganz neue Emotionen ins Spiel. Die Außenseite ist auch Veränderungen unterworfen. Einige davon wurden schon genannt als Liebeshandlungen, die Erwachsenen vorbehalten sind oder typischerweise erst dann auftreten und noch nicht in der Jugend: ein gemeinsamer Haushalt, gemeinsame Fürsorge für ein Kind, finanzielle und rechtliche Verwobenheit (Burkart 2018). Auch die externen Bedingungen ändern sich und haben wohl Einfluss auf die Innen- und Außenseite romantischer Liebe. Die Stressoren von Armut und Arbeitslosigkeit treten oftmals erst auf, wenn der Schutzraum der Jugend verlassen wird, die größere Eigenverantwortung im Berufsleben, neue Erwartungshaltungen von dritten, neue Anerkennungsmuster, die sich an Erfolg und Leistung und ihren materiellen und symbolischen Dingen orientieren, wie eine Beförderung, Macht, Einkommen, Haus oder Auto. Diese Dinge sind auch schon in der Jugend präsent und treten nicht plötzlich ins Leben des Menschen in kapitalis- 
tischen Geldgesellschaften. Mitunter kommt es zu einer Verlängerung eines quasijugendlichen Schutzraums für die Zeiten des Studiums, währenddessen die materielle Absicherung noch durch die Eltern erfolgt oder der junge Erwachsene noch zu Hause wohnt.

Mit dem Ausblick auf eine künftig mögliche romantische Liebe im Erwachsenenleben ist zweierlei gesagt. Erstens gibt es eine Verbindung zwischen dem, was als Jugendliche erlebt und erlernt wurde, und dem, was im Erwachsenenleben geschieht (sowohl auf der Innen- als auch der Außenseite) (Shulman und Kipnis 2001). Wenn man also der gut begründeten Meinung ist, dass romantische Liebe während des Erwachsenenlebens Teil eines guten Lebens ist, dann wird man nicht umhin können, ihre Quellen in der Jugend mitdenken zu müssen, selbst wenn man in Frage stellt, dass romantische Liebe Teil einer guten Jugend ist. Welche Erlebnisse und Lernprozesse machen es wahrscheinlicher, eine solche reife Liebe im Erwachsenenleben erfahren, reflektieren und leben zu können? Das sind psychologische und auch sozialwissenschaftliche Fragen und die Forschung zeigt, dass es eine solche Verbindung gibt (Larson u. a. 2002; Collins 2003). Die Frage, wie romantische Liebesbeziehungen während der Jugend das spätere Leben beeinflussen, ist aus methodischen Gründen nicht einfach zu beantworten. Eine Metastudie fasst die Gründe dafür zusammen (Manning u. a. 2008): Es liegt aus Sicht der psychologischen Bindungstheorie, die recht weit verbreitet ist, nahe, dass vorangegangene Bindungserfahrungen spätere beeinflussen. In romantischen Liebesbeziehungen, werden bestimmte Fähigkeiten ausgebildet und eingeübt, die im Kontext späterer Beziehungen eingesetzt oder angepasst werden können. Jugendliche entwickeln aber nicht nur eine Reihe an Fähigkeiten, sondern auch Ideale und Erwartungen von erwachsenen Beziehungen und wie diese ausgestaltet werden sollten und sie gehen damit an ihre eigenen Liebesbeziehungen heran. Damit ist keine starke Kausalität gefordert und auch nicht ausgeschlossen, dass es unkontrollierbare Faktoren gibt, die für das romantische Liebesleben eine wichtige Rolle spielen. Es genügt, wenn man mit guten Gründen annehmen kann, dass jugendliche Lernprozesse oder Erfahrungen einen Einfluss darauf haben, ob und wie man als Erwachsener romantische Liebe realisieren kann.

Zweitens, selbst wenn es keine solche Verbindung zwischen jugendlicher und erwachsener Liebe gäbe, erstere sich also nicht auf letztere auswirken würde, ist es plausibel anzunehmen, dass Jugendliche auf diese reife romantische Liebe vorbereitet werden sollten, so wie sie auch auf andere wesentliche Dinge des Erwachsenenlebens vorbereitet werden sollten, um damit gut umgehen zu können. Natürlich sind dieser Vorbereitung und diesen Lernprozessen Grenzen gesetzt, wie sie überall anzutreffen sind. Wie sich Langzeitbeziehungen anfühlen und wie gemeinsame Lebensplanung mit Kindern gelingen kann, kann nicht einfach erlernt werden, sondern bedarf auch des Erlebens und des Hineinwachsens in neue Lebensumstände und -abschnitte. Dennoch spielen Wissen und Informationen ebenso wie emotionale und kognitive Lernprozesse, die unterstützt und angeleitet werden können, eine wichtige Rolle. Ein vielleicht etwas abgegriffenes Beispiel sind patriarchale Rollenbilder oder kommunikative Ressourcen, um die eigenen Gefühle verstehen und kommunizieren zu können. Wer glaubt, dass dadurch der romantische Liebe ihre Magie genommen wird und diese einfach ein überwältigendes, natürliches Geschehen ist, welches man zulassen muss, verkennt den großen Einfluss sozialer Normen und Praktiken und 
eingeübter Verhaltensmuster. Ebenso klar ist aber natürlich auch, dass solche Lernprozesse in der Jugend keine Garantien abgeben können, dass im Erwachsenleben romantische Liebe gelingt, alleine schon weil dazu, in den allermeisten Fällen, eine zweite oder vielleicht sogar mehr Personen gehören, die ihre eigenen Wünsche und Vorstellungen, Fähigkeiten und Beschränkungen mitbringen. Was hier als Gelingen der romantischen Liebe im Erwachsenenleben - wie auch während der Jugend $\mathrm{zu}$ verstehen ist, kann nach unterschiedlichen Maßstäben bewertet werden: es gibt soziale und kulturelle Maßstäbe (etwa Heirat, Familiengründung) genauso wie individuelle Präferenzen. Denkbar wäre aber auch ein normatives Verständnis dessen, was gute romantische Liebe ausmacht, wobei hier unterschiedliche Antworten in der Philosophie gegeben werden.

\section{Die Ermöglichung und Regulierung jugendlicher Liebe durch die Eltern und den Staat}

Wenn also das Erleben und Erlernen romantischer Liebe in der Jugend eine wichtige Funktion für ein gutes Leben als Jugendliche und dann später als Erwachsener haben, dann kann nun danach gefragt werden, welche normativen Implikationen im Sinne von Rechten und Pflichten sich daraus ableiten lassen. Rechte und Pflichten von Jugendlichen betreffen immer mehrere Akteure, da sie in staatliche Institutionen eingebunden und speziellen rechtlichen Regelungen unterworfen sind und zumeist noch in einer Familie leben, in der erwachsene Erziehungsberechtigte mit speziellen Rechten und Pflichten gegenüber den Jugendlichen ausgestattet sind. Jugendliche romantische Liebe wird also in vielen Belangen stärker kontrolliert und reguliert als Liebe im Erwachsenenleben, was durch die Schutzfunktion der Eltern und des Staates gerechtfertigt ist, aber diese Regulierung und Kontrolle bedarf der Abwägung mit den legitimen Interessen der Jugendlichen. Ich beschränke mich in diesem Abschnitt auf die Rechte und Pflichten der Eltern und des Staates und lasse andere wichtige Akteure außer Acht, wie zum Beispiel die Medien, die durchaus Einfluss darauf haben, was Jugendliche über romantische Liebe wissen und wie sie in Liebesbeziehungen handeln.

Was kann nun also legitim von den Eltern und dem Staat und seinen Institutionen verlangt werden? Welche Rechte und Pflichten haben sie gegenüber der romantischen Liebe während der Jugend? Ich kann hier nur versuchen, die Diskussion um die Findung von konkreten Antworten zu strukturieren und wichtige Gesichtspunkte zu benennen. Konkrete Antworten können dann nur auf konkrete Fragen gegeben werden (z.B. ob und wann Jugendliche ein Recht haben, zu heiraten; ob Eltern [aus kulturellen oder religiösen Gründen] ein Recht haben, Einfluss auf die Partnerwahl zu nehmen; oder ob der Staat ein Recht hat, bestimmte Ausprägungen der Jugendsexualität wie Sexting unter Verbot zu stellen). Ich bringe sechs Punkte vor, um das komplexe Feld zu strukturieren.

Erstens ist es plausibel anzunehmen, dass wenn romantische Liebe in der Jugend ein so wichtiger Teil einer guten Jugend ist, dass Jugendliche ein prinzipielles Recht darauf haben, sie zu erleben, sofern dem nicht höherwertige Güter entgegen stehen. Das betrifft die Innen- wie auch die Außenseite der romantischen Liebe. 
Diese höherwertigen Güter sind eigens zu explizieren und zu rechtfertigen. Plausibel scheint hier die Annahme, dass Jugendliche sich und andere nicht übermäßig gefährden sollen und diese Gefährdung mit Blick auf ihr aktuelles und ihr künftiges Wohlergehen bewertet werden muss. Gänzlich ohne Risiko wird Liebeshandeln, ja die gesamte jugendliche Lebenswelt, nicht zu haben sein. Alleine das Risiko unerfüllter und unerwiderter Liebe ist groß und Liebeskummer erzeugt echte Schmerzen und kann zu selbstverletzendem Handeln führen (Price u. a. 2016). Das sollte nicht leichtfertig abgetan oder heruntergespielt werden. Ein Wert der romantische Liebe ergibt sich auch daraus, dass diese und Autonomie in einem engen Verhältnis stehen. Wen man liebt, gehört zum ureigenen privaten Lebensbereich. Man kann sich zwar nur schwerlich aussuchen, wen man liebt, aber es gehört zur eigenen Freiheit, dieser Liebe Handlungen folgen zu lassen, eine Beziehung zu beginnen und diese zu gestalten. Diese konkrete Ausgestaltung der romantischen Liebe, also die Art und Weise der Liebesbeziehung, die man führt, kann dann sicherlich wiederum autonomiefördernder oder autonomiebegrenzender erfolgen. Es gibt hier auch empirische Hinweise darauf, dass Autonomie für das Gelingen einer romantischen Liebesbeziehung wichtig ist (J. R. Anderson 2020).

Wenn also, zweitens, davon ausgegangen werden kann, dass jugendliche Autonomie anders als bei Kindern stärker zu berücksichtigen ist, dann überträgt sich dies sicherlich auf einen solch intimen und privaten Bereich wie das eigene romantische Liebesleben. Daraus folgen gewisse Ansprüche auf eine jugendliche Privatsphäre, die zu respektieren sind. Für die Eltern und den Staat sind der Wert und die Grenzen der romantischen Liebe aus je eigener Perspektive zu sehen, da sie gegenüber dem einzelnen Jugendlichen andere Rollen einnehmen. Weder kann es Aufgabe des Staates sein, Elternschaft im Sinne personaler Nahebeziehung und eines Mikromanagements des Familienlebens zu übernehmen, noch haben Eltern die Aufgaben des Staates zu übernehmen, allgemeinverbindliche Regeln für alle Jugendlichen aufzustellen und Chancen gleich zu verteilen. Eltern und Staat sind unterschiedliche Akteure, die unterschiedliche Motivationen, Ressourcen und Verpflichtungen haben. Die Eltern sind ihren Kindern gegenüber für unmittelbare Fürsorge, Liebe, Verständnis, Vertrauen und Unterstützung zuständig, die nur teilweise durch den Staat, seine Institutionen und Repräsentanten substituiert werden können. In der Regel kennen Eltern ihre Kinder auch besser als der Staat, da durch das gemeinsame Wohnen und Leben vielfältige Erfahrungen gemacht wurden. Im Fall der Entwicklung, des Lernens und Erlebens von romantischer Liebe sind gerade diese, nur in Nahebeziehungen kultivierbaren Güter besonders relevant, obwohl man wahrscheinlich keine empirischen Forschungen zu zitieren braucht, um zu wissen, dass Eltern-Kind-Beziehungen gerade in der Pubertät und gerade hinsichtlich solcher emotionalen und intimen Angelegenheiten wie der romantischen Liebe nicht immer ideal aufgestellt sind (Weymouth und Buehler 2016). Es gehört wohl auch dazu, dass viele Jugendliche hier eigene Erlebensräume für sich schaffen wollen, die den Eltern nicht zur Kenntnis gebracht werden. Auch für die gelingende Eltern-Kind-Beziehung während der Pubertät gibt es aber wissenschaftlich fundierte Konzepte, die vielen Eltern unbekannt bleiben, da es staatlicherseits nur sehr wenige Bemühungen gibt, Eltern in ihrer Elternrolle in dieser Hinsicht zu unterstützen und ihnen Wissen zukommen zu lassen (Ponzetti 2016). 
Drittens ist neben dem Respekt der Privatsphäre der Jugendlichen, also der Ermöglichung des Setzens von Liebeshandlungen unterschiedlichster Art und Weise, die Rolle der Eltern insbesondere für die Lern- und Reflexionsprozesse wichtig wobei dieses Recht auf Privatsphäre von Jugendlichen nicht uneingeschränkt gilt, sondern in Grenzen, die die Eltern auf Basis der Reife und anderer Faktoren, zu treffen haben. Es ist zwar so, dass es für viele Eltern selbst nicht einfach ist, über romantische Liebe, Begehren, Erotik oder Sex mit ihren jugendlichen Kindern zu sprechen und in der Ausbildung dieser Fähigkeit Unterstützung benötigen (Pariera und Brody 2018; Martino u. a. 2008), dennoch sind sie ein wichtiger Gesprächspartner und eine relevante Informationsquelle für ihre Kinder; selbst wenn sie nicht darüber kommunizieren, sendet dies eine Botschaft und die Jugendlichen sehen jedenfalls wie ihre Eltern ihr Liebesleben gestalten. Die gelungene Kommunikation zwischen Eltern und Jugendlichen ist etwas, das Eltern sich aneignen sollten, wenn sie gute Eltern sein wollen und der Staat kann hier unterstützen, indem er Informationsmaterial, aber auch Schulungen usw. bereitstellt. Die Eltern sind insbesondere dann in der Verantwortung, um für ihre Kinder in Phasen der Trauer, Verunsicherung und Verletzung, die im Rahmen vieler jugendlich-romantischer Liebeserfahrungen durchlaufen werden, unterstützend da zu sein.

Viertens ist der Staat verantwortlich, für gelungene Rahmenbedingungen zu sorgen und er ist, anders als die Eltern, allen Jugendlichen gleichermaßen verpflichtet. Diese Verpflichtungen lassen sich in kind- bzw. jugendspezifischen Theorien der Gerechtigkeit ausbuchstabieren (Schweiger und Graf 2015). Romantische Liebe während der Jugend ist nicht unmittelbar der Verteilung durch den Staat zugänglich, da es sich nicht um ein entsprechendes materielles Gut handelt, aber der Staat hat dennoch mehrere Interventionsmöglichkeiten, die beeinflussen, ob und wie romantische Liebe während der Jugend möglich ist, und daraus folgend hat er diese Interventionen auch gerecht zu setzen. Anca Gheaus etwa hat argumentiert, dass Liebe ein Gut der Gerechtigkeit ist, insofern diese für ein gutes Leben wichtig ist und der Staat ihre Verteilung beeinflussen kann (Gheaus 2017). Dies gilt auch für Jugendliche. Eine soziale Ordnung, die nur bestimmten Jugendlichen die Möglichkeit zur romantischen Liebesbeziehung gibt oder wichtige Teilgüter unfair verteilt, wie zum Beispiel den Zugang zu Wissen über romantische Liebe oder den Zugang zu Verhütungsmitteln, ist nicht legitimierbar. Ebenso problematisch sind soziale Arrangements, die die romantische Liebe für Jugendliche zerstören wie etwa eine rape culture, in der Mädchen massenhaft und strukturell Opfer sexueller Gewalt und hierdurch traumatisiert werden.

Der Staat hat sich also damit zu befassen, welchen Einfluss soziale, ökonomische, politische oder rechtliche Faktoren auf das Erleben und Erlernen romantischer Liebe während der Jugend haben und ob und inwieweit er diese beseitigen kann. Der sozioökonomische Status sollte zum Beispiel nicht determinieren, ob Jugendliche einen Zugang zu und Wissen über Verhütungsmittel haben, da dadurch das romantische Liebesleben - nicht nur dieses aber auch dieses - beeinflusst und das Risiko für die Übertragung von Krankheiten und ungewollte Schwangerschaften drastisch erhöht wird. Das schränkt die Autonomie dieser Jugendlichen stark ein und benachteiligt sie gegenüber besser gestellten Altersgenossen. Manche andere Faktoren liegen auf der Hand, die romantische Liebe direkt betreffen: so ist es zum Beispiel ungerecht, 
wenn der Staat eine Ordnung aufrechterhält, in der Mädchen sich ihre Liebespartner nicht aussuchen können, sondern von den Eltern verheiratet werden. Es ist auch ungerecht, wenn der Staat Gesetze oder auch nur soziale Normen unterstützt, die homosexuelle Liebesbeziehungen verbieten oder als unmoralisch verteufeln.

Fünftens hat der Staat weiterhin eine Schutzfunktion, die sich auch darauf auswirkt, was im Bereich der romantischen Liebe rechtlich erlaubt und was verboten sein sollte. Damit legt er Regeln oberhalb der Ebene der Eltern für alle Jugendlichen fest und beschreibt die Grenzen des Handlungsspielraums der Eltern. Es ist sinnvoll, unterschiedliche Arten der Begrenzung und Einschränkung jugendlicher Liebe zu unterscheiden: absolute Grenzen, die der Staat mittels Gesetzen durchsetzen sollte, wie im Bereich der Machtausnutzung in romantischen Beziehungen zwischen Lehrern und Schülern. Unterhalb dieser absoluten Grenzen, die auch jeweils auf ihre Adäquatheit geprüft werden müssen, gibt es ein weites Feld an Formen der romantischen Liebe und Beziehungen bzw. von Praktiken, die in romantischen Liebesbeziehungen stattfinden, die zwar mitunter moralisch problematisch erscheinen, aber kein Eingreifen des Staates rechtfertigen und in die Verantwortung der Eltern überlassen werden.

Dabei ist also auszuloten, inwieweit der Staat die Rechte von Jugendlichen gegenüber den Rechten ihrer Eltern und anderer Erziehungsberechtigter zu schützen und notfalls auch mit Staatsgewalt durchzusetzen hat. Das ist ein schwieriges Terrain, da der Eingriff ins Familienleben in vielen modernen Staaten als besonders heikel und die (kleinbürgerliche) Familie als Ideal gilt. Es wäre zu viel vorgenommen, wenn ich hier dieses Thema durchexerzieren würde. Der von mir präferierte Ansatz, der das Wohl und die Rechte von Jugendlichen in den Mittelpunkt stellt, sieht elterliche Rechte der Beschränkung jugendlicher Liebe gegenüber dem jugendlichen Interesse an romantischer Liebe als nachgeordnet an (Archard 2003). Das bedeutet, dass auch wenn die Familie ein prinzipiell schützenswerter Raum ist und Eltern eine wichtige Funktion für ihre Kinder einnehmen, so sind sie doch in ihrer Verfügungsgewalt aus moralischer Perspektive begrenzt werden sollten, um zwei Dinge zu erreichen: Einerseits die Forderung, dass alle Jugendliche möglichst gleiche Chancen der romantischen Liebe vorfinden sollten und andererseits, um im je eigenen Leben das Gut der romantischen Liebe erfahren zu können. Der erste Punkt bezieht sich darauf, dass unterschiedliche Erziehungsstile und Wertvorstellungen der Eltern dazu führen, dass Jugendliche gegenüber anderen übermäßig benachteiligt werden. Eltern haben also kein gutes Recht, ihre homosexuellen Kinder dahingehend zu indoktrinieren, dass Homosexualität moralisch falsch oder eine Sünde ist. Dadurch würden diese Jugendlichen benachteiligt, insofern es ihnen schwerfallen würde ihre eigene sexuelle Orientierung positiv annehmen zu können. Der zweite Punkte bezieht sich darauf, dass Jugendliche durch ihre Eltern nicht daran gehindert werden dürfen, romantische Liebe zu entdecken und zu leben, soweit dieses Liebeshandeln nicht ihr Wohlergehen gefährdet. Eltern haben also kein Recht, willkürlich Beziehungen zu verbieten oder die private Kommunikation ihrer jugendlichen Kinder vollständig zu überwachen. Der Staat kann hier nicht vollständige Gleichheit zwischen Jugendlichen erreichen oder anstreben, da dies nur ginge, wenn die Familie und die elterlichen Rechte prinzipiell aufgehoben werden würden, wogegen andere Gründe des Kindeswohl und der Selbstbestimmung von Eltern sprechen. Der Staat hat hier also Interventions- 
aufgaben, insofern er Eltern dazu erziehen und dabei unterstützen kann, dass sie die Rechte ihrer jugendlichen Kinder berücksichtigen und in gravierenden Fällen, kann er auch gesetzliche Regelungen erlassen. Elterliche Gewalt, sei sie physisch oder psychisch, zur Verhinderung von romantischen Liebesbeziehungen oder andere gravierende Eingriffe wie die Zwangsverheiratung von Jugendlichen, sind klare Fälle, wo staatliche Interventionen geboten sind.

Sechstens hat der Staat, weil er allen Jugendlichen gegenüber gleichermaßen verpflichtet ist, sich gerade darum zu kümmern, dass auch alle jene Jugendlichen, deren Eltern ihre Rolle nicht ausreichend wahrnehmen können oder wollen, in ihrer Entwicklung und ihren Lernprozessen unterstützt werden, also leicht zugängliche Bildungs- und Beratungsangebote vorfinden. Hier kommt auch die Schule als zentraler Ort jugendlicher Lebenswelt und staatlichen Zugriffs auf diese in den Blick. Die Diskussion um Sexualerziehung, die teils heftig zwischen besorgten Eltern auf der einen Seite und Lehrern, Schulen und Politikern auf der anderen Seite geführt wird, zeigt, dass es hier durchaus Bruchstellen und Konfliktpotentiale gibt, weil Eltern sehr bestimmte Vorstellungen darüber haben, was im Bereich der Liebe und Sexualität gelehrt werden sollte und was ihren eigenen Moralvorstellungen widerspricht (Drerup 2019). Auch wenn zur romantischen Liebe mehr gehört als Sex und Sexualunterricht daher auch andere Funktionen und Inhalte hat, ist nicht zu leugnen, dass auch in dieser Hinsicht Konflikte entstehen werden. Etwa wenn es darum geht, Wissen über unterschiedliche Konstellationen der romantischen Liebe (hetero-, homo- und bisexuelle Paarbeziehungen ebenso wie Polyamorie) in der Schule zu vermitteln, damit Jugendliche selbstbestimmt die eigenen Präferenzen verstehen lernen.

Insofern man jugendliche Autonomie ernst nimmt - und es gibt, wie gesagt, gute Gründe, das zu tun - ergeben sich auch unterschiedliche Interventionsmittel, die der Staat oder die Eltern nutzen können: Aufklärung und Bildung, Incentives und Nudging, milde Formen der Sanktionen durch die Eltern oder andere Institutionen. Ein Beispiel: Wie können Jugendliche dazu motiviert werden, Verhütungsmittel zu benutzen? Sex ist ein wichtiger Bestandteil romantischer Liebe und viele Jugendliche haben Sex als Teil ihres Liebeslebens, aber Sex birgt auch einige Gefahren, darunter Krankheiten und kann zu einer Schwangerschaft führen, was im jugendlichen Alter eine große Belastung sein kann. Man kann Jugendliche aufklären und zwar durch die Eltern und durch die Schule. Das kann verpflichtend sein oder es werden Anreize geschaffen, solche Lerneinheiten zu besuchen. Man kann Aufklärung über Medien verbreiten und dort mit anderen Inhalten oder Angeboten koppeln, damit sie auch wahrgenommen werden (etwa indem bekannte Musikerinnen als Testimonials fungieren). Man kann Kondome gratis in Schulen oder bei Jugendveranstaltungen verteilen. Jugendliche haben noch immer die Wahl, ob und wie sie verhüten, aber ihr Verhalten wird dadurch beeinflusst. Welche Interventionen, effektiv sind oder welche auch nicht gerechtfertigt wären - etwa an Jugendliche keine Kondome zu verkaufen, weil man glaubt, dann hätten sie keinen Sex - bedarf der Kombination aus empirischen und normativen Überlegungen.

Wichtig scheint mir jedoch ein Perspektivenwechsel, der romantische Liebe während der Jugend und alle was dazu gehört, insbesondere auch Sexualität, nicht nur als Gefahr zu sehen, sondern die positiven Potentiale jugendlicher Liebe und die mit 
ihr verbundenen Fähigkeiten des Vertrauens, der Fürsorge, der Romantik, des sexuellen Begehrens, der Partnerschaft und Hoffnung zu stärken und jungen Menschen dabei zu helfen, diese zu entwickeln und zu leben. Dafür ist es auch nötig, vor die Lebensphase zu blicken und den nochmals sensibleren Topos kindlicher Gefühlswelten inklusive Begehren und Sexualität zu thematisieren (Graf und Schweiger 2017; Brennan und Epp 2015). Die Fähigkeiten und das Wissen, welches für eine gelingende romantische Liebe in der Jugend benötigt werden, haben ihre Wurzeln schon früher - in der frühkindlichen Entwicklung und Bindung (Ahnert und Maywald 2004), die es erlaubt jene Fähigkeiten und Einstellungen (Selbstvertrauen, Vertrauen in andere, Selbstwert etc.) zu entwickeln, die dann für romantische Liebe wichtig werden.

\section{Schluss}

Zum Schluss will ich meine Argumente nochmals zusammenfassen und hiernach einige Lücken ansprechen, die mein Text hinterlässt, die es aber wert wären, philosophisch reflektiert zu werden. Ich habe dafür plädiert, dass romantische Liebe ein Teil einer guten Jugend ist. Das scheint trivial angesichts der Omnipräsenz jugendlicher Liebe und der hohen Wertschätzung, die ihr entgegengebracht wird, dennoch wurde dieses Gut in der Philosophie, die sich in den letzten Jahren immer stärker mit Fragen der Kindheit und Jugend beschäftigt, bislang ignoriert. Wichtig ist es jedoch, romantische Liebe nicht einfach als unkontrollierbares Gefühlsgeschehen zu thematisieren, sondern zu fragen, wie Jugendliche lernen können, mit ihren Emotionen und den damit verbunden Wünschen und Handlungen, den vielen Normen und Praktiken, die mit romantischer Liebe verbunden sind, gut umzugehen. (Fast) Alle Güter, auf die Jugendliche einen moralischen Anspruch haben, beinhalten solche Lernprozesse und nur allzu oft, wird dies übersehen. Zum Beispiel: Niemand wird bestreiten, dass es für Jugendliche moralisch wertvoll ist, gesund zu sein. Aber, wie der eigene Körper funktioniert, wie man sich gesund ernährt, welche Faktoren einen negativen Einfluss auf die Gesundheit haben, wo Gefahren lauern und wie sie sich davor schützen können etc. sind alles Wissensbestände und schließlich auch Praktiken, die erlernt und erfahren werden müssen. Jugendlichen das vorzuenthalten, raubt ihnen also wichtige Möglichkeiten und macht ihr Leben schließlich schlechter als es sein könnte. In diesem Sinne verstehe ich die Aufgabe der Eltern und des Staates, die beiden Akteure, die ich näher beleuchtet habe, nämlich, dass sie Jugendlichen diesen wichtigen Teil ihrer Lebenswelt, diesen wichtigen Teil ihres Lebens und ihres Aufwachsens, den sie selbst und andere schätzen, ermöglichen und Spielräume geben, ihnen die nötige Bildung geben, sie dafür respektieren, dass sie hier wichtige Entscheidungen für sich selbst treffen, aber auch vor den Gefahren schützen und sie unterstützen, wo sie Unterstützung benötigen. In den Details sind hier viele Fragen offen, die in einem Aufsatz alleine nicht beantwortet werden können.

Es wurde ausgespart, wie gelingende Liebesbeziehungen in der Jugend normativ definiert werden sollten und auch nur sehr wenig über die Unterschiede zwischen Jugendlichen und Erwachsenen aus Sicht unterschiedlicher Theorien der Liebe. Das ist nicht nur eine analytische Frage, sondern hat vielleicht auch normative Impli- 
kationen, da es nahelegt, welche Inhalte in den Lernprozessen verankert werden sollten. Einige Aspekte scheinen unstrittig, wie zum Beispiel Gewaltfreiheit oder Respekt der Autonomie der Partnerin und ihrer Wünsche und Grenzen. Sehr viele Dinge hinsichtlich der Geschlechterrollen, der sexuellen Aktivitäten etc. sind aber sicherlich hoch umstritten. Die Philosophie überlässt dieses Feld bislang fast vollständig der Pädagogik oder der Psychologie. Es geht aber auch um die moralischen Rechte und Pflichten von Jugendlichen, der Eltern und des Staates hier. Darf bzw. soll der Staat Sexualerziehung in den Schulen verpflichtend machen und was soll hier gelehrt und gelernt werden? Das ist im Kern (auch) eine ethische Frage.

Sehr wenig zur Sprache gekommen sind in diesem Beitrag auch die möglichen Subjekte der Liebe und die soziale Einrahmung unterschiedlicher Formen des Liebeshandelns. Auch wenn die Vermutung naheliegt, dass die Innen- und Außenseite der romantischen Liebe bei hetero-, homo- und bisexuellen Jugendlichen sehr ähnlich ist, so unterscheiden sie sich schon alleine dadurch, dass Homo- und Bisexualität vielerorts noch immer nicht (vollständig) akzeptiert ist und diese Jugendlichen mit besonderen Schwierigkeiten zu kämpfen haben, für die nötigen Lernprozesse adäquate Ressourcen und Unterstützung zu erfahren. Das Ausleben ihrer romantischen Liebe wird vielerorts auch noch immer nicht akzeptiert und sie haben mit Mobbing und Ausgrenzung - sowohl durch ihre Eltern, andere Jugendliche oder gar staatlichen Repräsentanten und Institutionen - zu kämpfen. Hier liegen unterschiedliche Gefahren für Ungerechtigkeiten, die es wert und sinnvoll wäre, auf ihre normativen Implikationen hin zu analysieren. Worüber ich leider auch nicht sehr viel sagen konnte, sind kulturelle und religiöse Traditionen und wie legitim ihre Einflussnahme auf das romantische Liebeshandeln von Jugendlichen ist. Schließlich ist es so, dass jugendliche romantische Liebe und insbesondere ihre Sexualität teilweise stark reguliert wird oder Versuche gemacht werden, einseitige und eventuell schädliche Konzepte von romantischer Liebe durchzusetzen. Zum Beispiel, die Frage: Dürfen homophobe Eltern ihre homosexuellen Kinder indoktrinieren und wenn nicht - Samantha Brennan und Colin Macleod (2017) haben so argumentiert - welche Konsequenzen könnte das haben?

Schließlich sei auch darauf hingewiesen, dass ich sehr wenig zu einer möglichen Sozialkritik der romantischen Liebe beitragen konnte. Eine solche Sozialkritik sollte die herrschenden Formen und Materialisierungen des romantischen Liebeshandeln analysieren und danach fragen, welchen sozialen Zwecken sie eigentlich dienen - der sozialen Regulierung und Kontrolle, der Verdinglichung und Kommerzialisierung etwa. Romantische Liebe ist mit einer ganzen Reihe an Ungleichheiten zwischen den Geschlechtern verbunden, die durch Stereotype und Skripte festgelegt werden - aber auch Ungleichheiten zwischen sozio-ökonomischen Schichten und Milieus. Jugendliche sind für solche Verwerfungen und Entfremdungen mindestens ebenso empfänglich wie Erwachsene, die durch die Sozialisation, die Medien und die Warenwelt der romantischen Liebe auf bestimmte soziale Normen und Praktiken festgelegt werden. Romantische Liebe ist eben nicht nur eine Emotion, sondern sie eignet sich gut dazu, um Produkte zu verkaufen und sie eignet sich auch gut dazu, Mädchen ihre Unterordnung und Jungen ihre Dominanz zuzuschreiben und $\mathrm{zu}$ verinnerlichen. Ein solcher Blick auf die Verwerfungen der romantischen Liebe würde aber auch den Blick eröffnen auf die sozialkritischen Potentiale romanti- 
scher Liebe selbst, also ihre Widerständigkeit gegen soziale Normen und Praktiken und ob sie Räume schaffen kann für Authentizität jenseits der Warenförmigkeit des Liebeshandelns im Kapitalismus.

Danksagung Ich habe zahlreiche hilfreiche Hinweise zur Überarbeitung dieses Textes bekommen und bedanke mich hierfür bei Alexander Bagattini, Johannes Drerup, Martin Hähnel, Thomas Grote, Michael Kühler und den beiden anonymen GutachterInnen der ZEMO.

Funding Open access funding provided by Paris Lodron University of Salzburg.

Open Access Dieser Artikel wird unter der Creative Commons Namensnennung 4.0 International Lizenz veröffentlicht, welche die Nutzung, Vervielfältigung, Bearbeitung, Verbreitung und Wiedergabe in jeglichem Medium und Format erlaubt, sofern Sie den/die ursprünglichen Autor(en) und die Quelle ordnungsgemäß nennen, einen Link zur Creative Commons Lizenz beifügen und angeben, ob Änderungen vorgenommen wurden.

Die in diesem Artikel enthaltenen Bilder und sonstiges Drittmaterial unterliegen ebenfalls der genannten Creative Commons Lizenz, sofern sich aus der Abbildungslegende nichts anderes ergibt. Sofern das betreffende Material nicht unter der genannten Creative Commons Lizenz steht und die betreffende Handlung nicht nach gesetzlichen Vorschriften erlaubt ist, ist für die oben aufgeführten Weiterverwendungen des Materials die Einwilligung des jeweiligen Rechteinhabers einzuholen.

Weitere Details zur Lizenz entnehmen Sie bitte der Lizenzinformation auf http://creativecommons.org/ licenses/by/4.0/deed.de.

\section{Literatur}

Abad, Jamie Pax. 2014. „Das Glück der Selbstverwirklichung“. Internationales Jahrbuch für philosophische Anthropologie 4 (1): 75-84. https://doi.org/10.1515/jbpa-2014-0106.

Ahnert, Lieselotte, und Jörg Maywald, Hrsg. 2004. Frühe Bindung: Entstehung und Entwicklung. München: Reinhardt.

Anderson, Jared R. 2020. „Inviting Autonomy Back to the Table: The Importance of Autonomy for Healthy Relationship Functioning“. Journal of Marital and Family Therapy 46 (1): 3-14. https://doi.org/10. 1111 jmft.12413.

Anderson, Joel, und Rutger Claassen. 2012. „Sailing Alone: Teenage Autonomy and Regimes of Childhood“. Law and Philosophy 31 (5): 495-522. https://doi.org/10.1007/s10982-012-9130-9.

Archard, David. 2003. Children, family, and the state. 1. Aufl. Live questions in ethics and moral philosophy. Aldershot / Burlington, VT: Ashgate.

Ben-Ze'ev, Aaron. 2004. Love Online: Emotions on the Internet. 1. Aufl. Cambridge: Cambridge University Press. https://doi.org/10.1017/CBO9780511489785.

Best, Joel, und Kathleen A. Bogle. 2014. Kids gone wild: from rainbow parties to sexting, understanding the hype over teen sex. 1. Aufl. New York, NY: New York University Press.

Betzler, Monika. 2011. „Erziehung zur Autonomie als Elternpflicht“. Deutsche Zeitschrift für Philosophie 59 (6): 937-53. https://doi.org/10.1524/dzph.2011.0074.

Bongardt, Daphne van de, Rongqin Yu, Maja Deković, und Wim H. J. Meeus. 2015. „Romantic Relationships and Sexuality in Adolescence and Young Adulthood: The Role of Parents, Peers, and Partners“. European Journal of Developmental Psychology 12 (5): 497-515. https://doi.org/10.1080/17405629. 2015.1068689 .

Brennan, Samantha, und Jennifer Epp. 2015. „Children's Rights, Well-Being, and Sexual Agency“. In The Nature of Children's Well-Being, herausgegeben von Alexander Bagattini und Colin Macleod, 1. Aufl., 9:227-46. Dordrecht: Springer. http://link.springer.com/10.1007/978-94-017-9252-3_14.

Brennan, Samantha, und Macleod, Colin. 2017. Fundamentally Incompetent: Homophobia, Religion, and the Right to Parent. In: Procreation, Parenthood, and Educational Rights: Ethical and Philosophical Issues, von Jaime Ahlberg, und Michael Cholbi. New York: Routledge, 1. Aufl., 230-245.

Burkart, Günter. 2018. Soziologie der Paarbeziehung: Eine Einführung. 1. Aufl. Wiesbaden: Springer VS. https://doi.org/10.1007/978-3-658-19405-5. 
Calmbach, Marc, Silke Borgstedt, Inga Borchard, Peter Martin Thomas, und Berthold Bodo Flaig. 2016. „Liebe und Partnerschaft“. In Wie ticken Jugendliche 2016?, von Marc Calmbach, Silke Borgstedt, Inga Borchard, Peter Martin Thomas, und Berthold Bodo Flaig, 1. Aufl., 303-33. Wiesbaden: Springer. https://doi.org/10.1007/978-3-658-12533-2_7.

Collins, W. Andrew. 2003. „More than Myth: The Developmental Significance of Romantic Relationships During Adolescence“. Journal of Research on Adolescence 13 (1): 1-24. https://doi.org/10.1111/ 1532-7795.1301001.

Collins, W. Andrew, Deborah P. Welsh, und Wyndol Furman. 2009. „Adolescent Romantic Relationships“. Annual Review of Psychology 60 (1): 631-52. https://doi.org/10.1146/annurev.psych.60. 110707.163459

Cook, Sinead M.C., und Sharon T. Cameron. 2015. „Social Issues of Teenage Pregnancy“. Obstetrics, Gynaecology \& Reproductive Medicine 25 (9): 243-48. https://doi.org/10.1016/j.ogrm.2015.06.001.

Dekker, Arne, und Thula Koops. 2017. „Sexting als Risiko?: Zum konsensuellen und nichtkonsensuellen Versand persönlicher erotischer Fotos mittels digitaler Medien“. Bundesgesundheitsblatt - Gesundheitsforschung - Gesundheitsschutz 60 (9): 1034-39. https://doi.org/10.1007/s00103-017-2595-9.

Drerup, Johannes. 2019. „Sexualerziehung, staatliche Neutralität und der Wert der Vielfalt“. In Handbuch Philosophie der Kindheit, herausgegeben von Johannes Drerup und Gottfried Schweiger, 1. Aufl., 430-37. Stuttgart: J.B. Metzler. https://doi.org/10.1007/978-3-476-04745-8_54.

Drury, Kate Mills, und William M. Bukowski. 2013. „Sexual Development“. In Handbook of Child and Adolescent Sexuality, 115-44. Oxford: Academis Press. http://linkinghub.elsevier.com/retrieve/pii/ B9780123877598000052.

Fenner, Dagmar. 2015. „Zwischen Selbstverwirklichung und Selbsttranszendenz - Menschliche Selbstentwürfe und die ethische Frage nach dem Guten“. In Was ist der Mensch?, herausgegeben von Mathias Lindenau und Marcel Meier Kressig. Bielefeld: transcript Verlag. https://doi.org/10.14361/ 9783839430323-003.

Franklin-Hall, Andrew. 2013. „On Becoming an Adult: Autonomy and the Moral Relevance of Life's Stages“. The Philosophical Quarterly 63 (251): 223-47. https://doi.org/10.1111/1467-9213.12014.

Fricker, Miranda. 2007. Epistemic injustice: power and the ethics of knowing. 1. Aufl. Oxford / New York, NY: Oxford University Press.

Fromm, Erich. 2012. Die Kunst des Liebens. 70. Aufl. Ullstein 36784. Berlin: Ullstein.

Gallie, W. B. 1956. Essentially Contested Concepts. In: Proceedings of the Aristotelian Society 56: $167-198$.

Gheaus, Anca. 2017. „Love and Justice: a Paradox?“ Canadian Journal of Philosophy 47 (6): $739-59$. https://doi.org/10.1080/00455091.2017.1319656.

Giesinger, Johannes. 2017. „Wahlrecht für Kinder? Politische Initiation und der Status der Kindheit“. Archiv für Rechts- und Sozialphilosophie 103 (4): 456-69. https://doi.org/10.25162/ARSP-2017-0247.

Gill, Rosalind. 2012. „Media, Empowerment and the 'Sexualization of Culture' Debates“. Sex Roles 66 (11-12): 736-45. https://doi.org/10.1007/s11199-011-0107-1.

Graf, Gunter, und Gottfried Schweiger. 2017. Ethics and the Endangerment of Children's Bodies. Basingstoke: Palgrave Macmillan.

Grau, Christopher, und Aaron Smuts, Hrsg. 2017. The Oxford Handbook of Philosophy of Love. 1. Aufl. Oxford: Oxford University Press. https://doi.org/10.1093/oxfordhb/9780199395729.001.0001.

Gregoratto, Federica. 2017. „Love Is a Losing Game: Power and Exploitation in Romantic Relationships“. Journal of Political Power 10 (3): 326-41. https://doi.org/10.1080/2158379X.2017.1382172.

Helmer, Janet, Kate Senior, Belinda Davison, und Andrew Vodic. 2015. „Improving Sexual Health for Young People: Making Sexuality Education a Priority“. Sex Education 15 (2): 158-71. https://doi. org/10.1080/14681811.2014.989201.

Honneth, Axel. 1992. Kampf um Anerkennung: Zur moralischen Grammatik sozialer Konflikte. 1129. Frankfurt, Main: Suhrkamp.

Honneth, Axel. 2002. „Organisierte Selbstverwirklichung. Paradoxien der Individualisierung“. In Befreiung aus der Mündigkeit. Paradoxien des gegenwärtigen Kapitalismus, herausgegeben von Axel Honneth, 1. Aufl., 141-58. Frankfurter Beiträge zur Soziologie und Sozialphilosophie 1. Frankfurt am Main / New York, NY: Campus.

Illouz, Eva. 2007. Gefühle in Zeiten des Kapitalismus: Frankfurter Adorno-Vorlesungen 2004, Institut für Sozialforschung an der Johann-Wolfgang-Goethe-Universität, Frankfurt am Main. 1. Aufl. Frankfurt am Main: Suhrkamp.

Illouz, Eva. 2009. Der Konsum der Romantik: Liebe und die kulturellen Widersprüche des Kapitalismus. 1. Aufl. Frankfurt am Main: Suhrkamp. 
Jaeggi, Rahel. 2005. Entfremdung. Zur Aktualität eines sozialphilosophischen Problems. 1. Aufl. Frankfurter Beiträge zur Soziologie und Sozialphilosophie 13. Frankfurt am Main / New York, NY: Campus.

Jewell, Jennifer A., und Christia Spears Brown. 2013. ,Sexting, Catcalls, and Butt Slaps: How Gender Stereotypes and Perceived Group Norms Predict Sexualized Behavior“. Sex Roles 69 (11-12): 594-604. https://doi.org/10.1007/s11199-013-0320-1.

Khader, Serene J. 2011. Adaptive Preferences and Women's Empowerment. 1. Aufl. Oxford / New York, NY: Oxford University Press.

Krebs, Angelika. 2001. Arbeit und Liebe. Die philosophischen Grundlagen sozialer Gerechtigkeit. 1. Aufl. Frankfurt am Main: Suhrkamp.

Kühler, Michael. 2019. „Romantische Liebe und die Freiheit zu gehen“. In Die Freiheit zu gehen, herausgegeben von Simone Dietz, Hannes Foth, und Svenja Wiertz, 1. Aufl., 195-222. Wiesbaden: Springer VS. https://doi.org/10.1007/978-3-658-26668-4_8.

Larson, Reed W., Suzanne Wilson, B. Bradford Brown, Frank F. Furstenberg, Jr., und Suman Verma. 2002. „Changes in Adolescents' Interpersonal Experiences: Are They Being Prepared for Adult Relationships in the Twenty-First Century?" Journal of Research on Adolescence 12 (1): 31-68. https://doi. org/10.1111/1532-7795.00024.

Lehrer, Keith. 1997. „Love and Autonomy“. In Love Analyzed, herausgegeben von Roger E Lamb, 1. Aufl., 107-21. Boulder, CO: Westview Press.

Lindholm, Charles. 2006. „Romantic Love and Anthropology“. Etnofoor 19 (1): 5-21.

Mackenzie, Catriona, Wendy Rogers, und Susan Dodds. 2014. „Introduction: What Is Vulnerability and Why Does It Matter for Moral Theory?" In Vulnerability: New essays in ethics and feminist philosophy, herausgegeben von Catriona Mackenzie, Wendy Rogers, und Susan Dodds, 1-29. New York, NY: Oxford University Press.

Macleod, Colin. 2017. „Doctrinal Vulnerability and the Authority of Children's Voices“. In Vulnerability, Autonomy and Applied Ethics, herausgegeben von Christine Straehle, 171-84. New York, NY: Routledge.

Manning, Wendy D., Peggy C. Giordano, und Monica A. Longmore. 2008. „Adolescent Dating Relationships: Implications for Understanding Adult Unions“. Working Paper Series 2008-08. Bowling Green, OH: Bowling Green State University. The Center for Family and Demographic Research. http://www.bgsu.edu/content/dam/BGSU/college-of-arts-and-sciences/center-for-familyand-demographic-research/documents/working-papers/2008/CFDR-Working-Papers-2008-08Adolescent-Dating-Relationships-Implications-for-Understanding-Adult-Intimate-Unions.pdf

Martino, Steven C., Marc N. Elliott, Rosalie Corona, David E. Kanouse, und Mark A. Schuster. 2008. „Beyond the "Big Talk": The Roles of Breadth and Repetition in Parent-Adolescent Communication About Sexual Topics“. Pediatrics 121 (3): e612-18. https://doi.org/10.1542/peds.2007-2156.

Maurer, Christian, Tony Milligan, und Kamila Pacovská, Hrsg. 2014. Love and Its Objects. 1. Aufl. London: Palgrave Macmillan. https://doi.org/10.1057/9781137383310.

Merk, Kurt-Peter. 2009. „Das Wahlrecht von Geburt an und seine politische Bedeutung“. Diskurs Kindheits- und Jugendforschung / Discourse. Journal of Childhood and Adolescence Research 4: 525-38.

Mills, Claudia. 2003. „The Child's Right to an Open Future?“ Journal of Social Philosophy 34 (4): 499-509. https://doi.org/10.1111/1467-9833.00197.

Neumann, Eva. 2002. „Die Paarbeziehung Erwachsener und Erinnerungen an die Eltern- Kind-Beziehung - Eine Untersuchung zur Kontinuität von Bindung“. Zeitschrift für Familienforschung, Band 14, Nr 3: 234-56.

Pariera, Katrina L., und Evan Brody. 2018. „“Talk More About It”: Emerging Adults’ Attitudes About How and When Parents Should Talk About Sex“. Sexuality Research and Social Policy 15 (2): 219-29. https://doi.org/10.1007/s13178-017-0314-9.

Pettit, Philip. 2015. The robust demands of the good: ethics with attachment, virtue, and respect. 1. Aufl. Uehiro series in practical ethics. Oxford: Oxford University Press.

Pismenny, Arina, und Jesse Prinz. 2017. „Is Love an Emotion?“ In The Oxford Handbook of Philosophy of Love, herausgegeben von Christopher Grau und Aaron Smuts, 1. Aufl. Oxford: Oxford University Press. https://doi.org/10.1093/oxfordhb/9780199395729.001.0001.

Ponzetti, James J., Hrsg. 2016. Evidence-based parenting education: a global perspective. 1. Aufl. Textbooks in family studies. New York, NY: Routledge.

Price, Megan, Leanne Hides, Wendell Cockshaw, Aleksandra Staneva, und Stoyan Stoyanov. 2016. „Young Love: Romantic Concerns and Associated Mental Health Issues among Adolescent Help-Seekers“. Behavioral Sciences 6 (2): 9. https://doi.org/10.3390/bs6020009. 
Sawyer, Susan M, Peter S Azzopardi, Dakshitha Wickremarathne, und George C Patton. 2018. ,The Age of Adolescence“. The Lancet Child \& Adolescent Health 2 (3): 223-28. https://doi.org/10.1016/S23524642(18)30022-1.

Schweiger, Gottfried, und Gunter Graf. 2015. A Philosophical Examination of Social Justice and Child Poverty. 1. Aufl. Basingstoke: Palgrave Macmillan. http://www.palgraveconnect.com/doifinder/10. $1057 / 9781137426024$.

Schweiger, Gottfried, und Gunter Graf. 2017. „Ethics and the dynamic vulnerability of children“. Les Ateliers de l'éthique/The Ethics Forum 12 (2-3): 243-261. https://doi.org/10.7202/1051284ar.

Selman, Peter. 2003. „Scapegoating and Moral Panics: Teenage Pregnancy in Britain and the United States“. In Families and the State, herausgegeben von Sarah Cunningham-Burley und Lynn Jamieson, 1. Aufl., 159-86. London: Palgrave Macmillan. https://doi.org/10.1057/9780230522831_9.

Shulman, Shmuel, und Offer Kipnis. 2001. „Adolescent Romantic Relationships: A Look from the Future“. Journal of Adolescence 24 (3): 337-51. https://doi.org/10.1006/jado.2001.0409.

Stronski, Susanne M. 2018. „Körperbild bei Mädchen und Jungen in der Pubertät“. In Jugendmedizin, herausgegeben von Bernhard Stier, Nikolaus Weissenrieder, und Karl Otfried Schwab, 1. Aufl., 25-29. Berlin / Heidelberg: Springer. https://doi.org/10.1007/978-3-662-52783-2_3.

Walter, Henrik. 2004. „Liebe und Lust. Ein intimes Verhältnis und seine neurobiolgischen Grundlagen“. In Natur und Theorie der Emotion, herausgegeben von Achim Stephan und Henrik Walter, 2. Aufl., 75-111. Paderborn: Mentis.

Weymouth, Bridget B., und Cheryl Buehler. 2016. „Adolescent and Parental Contributions to Parent-Adolescent Hostility Across Early Adolescence“. Journal of Youth and Adolescence 45 (4): 713-29. https://doi.org/10.1007/s10964-015-0348-3. 